\title{
Scanning Laser Ophthalmoscopy and Angiography With a Wide-Field Contact Lens System
}

Giovanni Staurenghi, MD; Francesco Viola, MD; Martin A. Mainster, PhD, MD;

Raymond D. Graham, ASc; Peter G. Harrington, BSc

Objective: To perform fluorescein and indocyanine green angiography for large or peripheral chorioretinal structures using a contact lens system that provides a 5-fold increase in the field of view of a confocal scanning laser ophthalmoscope (SLO).

Methods: Separate handheld contact and noncontact ophthalmoscopic lenses were manually aligned with the optical axis of a confocal SLO to demonstrate the feasibility of wide-field SLO angiography. An integrated, widefield contact lens system was then designed and constructed to increase the SLO's $10^{\circ}, 20^{\circ}$, and $30^{\circ}$ imaging fields to $50^{\circ}, 100^{\circ}$, and $150^{\circ}$, respectively.

Results: Simultaneous fluorescein and indocyanine green angiography was performed with the integrated, widefield contact lens system for more than 50 patients with disorders that affect their peripheral retina and choroid. Retinal and choroidal abnormalities, including neovas- cularization and capillary nonperfusion, are easily detected and documented well beyond the range of conventional fundus cameras and SLOs. Peripheral retinal and choroidal hemodynamics can be readily observed and recorded.

Conclusions: A confocal SLO has adequate resolution for clinically useful reflectance and angiographic imaging even when its field size is increased 5-fold by a widefield contact lens system. Dynamic and static wide-field angiography can be performed without the limitations of manual or computer-automated photomontages. Peripheral retinal conditions can be studied and recorded to confirm observations from indirect ophthalmoscopy and to facilitate retinal photocoagulation and vitreoretinal surgery.

Arch Ophthalmol. 2005;123:244-252
Author Affiliations: University Eye Clinic, University of Brescia, Brescia, Italy (Drs Staurenghi and Viola); Department of Ophthalmology, University of Kansas Medical Center, Kansas City (Dr Mainster); and Ocular Instruments Inc, Bellevue, Wash (Messrs Graham and Harrington).

Financial Disclosure: Dr Staurenghi and Messrs Graham and Harrington hold a patent on the Ocular Staurenghi 230 SLO Retina Lens.

Dr Mainster acts as a consultant to and Messrs Harrington and Graham are employees of Ocular Instruments Inc, which manufactures the Ocular Staurenghi 230 SLO Retina Lens.

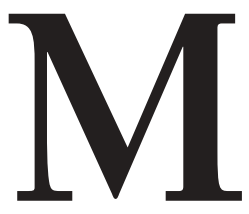

ODERN CONFOCAL scanning laser ophthalmoscopy (SLO) produces dynamic high-resolution retinal images at lower retinal irradiances than conventional fundus photography. ${ }^{1}$ Indocyanine green (ICG) and fluorescein angiography can be performed simultaneously. ${ }^{2,3}$ Typical $30^{\circ}$ SLO images are excellent for most retinal applications but inadequate for recording chorioretinal findings that are large or located well anterior to the ocular equator.

An accessory contact or noncontact ophthalmoscopy lens can significantly enlarge a conventional fundus camera's $20^{\circ}$ to $60^{\circ}$ angiographic field. ${ }^{4-6}$ Specialized wide-field contact camera systems provide angiographic fields that range from $80^{\circ}$ to $148^{\circ} .7,8$ An Equator-plus (MIRA Inc, Uxbridge, Mass) camera contact lens element ${ }^{7}$ was used to demonstrate the feasibility of contact lens expansion of nonconfocal SLO imaging fields. ${ }^{9}$ A $30-$ diopter (D) noncontact ophthalmoscopy lens was used to expand the imaging field of a confocal SLO to $70^{\circ}$ in a study of peripheral choroidal circulation. ${ }^{10}$ We report the clinical trials of a wide-field contact lens system that increases the imaging field of a confocal SLO up to $150^{\circ}$.

\section{METHODS}

We used a Heidelberg retina angiograph (HRA; Heidelberg Engineering GmbH, Dossenheim, Germany) confocal SLO with 4 laser wavelengths: $488 \mathrm{~nm}$ for fluorescein angiography, $514 \mathrm{~nm}$ for green (red-free) reflectance imaging, $795 \mathrm{~nm}$ for ICG angiography, and $835 \mathrm{~nm}$ for infrared reflectance imaging. All aspects of this investigation were approved by the Institutional Review Board of the Department of Surgical Specialties, Radiologic Sciences and Legal Medicine of the University of Brescia, Brescia, Italy.

We established the feasibility of wide-field confocal SLO imaging by examining patients while holding a separate 28-D noncontact and a wide-field contact ophthalmoscopy lens with 
their optical axes aligned parallel to the axis of an HRA SLO. Initial images were useful, but the difficulty of maintaining manual lens alignment prompted us to design and construct an integrated, multielement, wide-field contact lens system (Ocular Staurenghi 230 SLO Retina Lens; Ocular Instruments Inc, Bellevue, Wash). All design calculations for the lens system were performed with the OSLO ray tracing program (release 6.1; Lambda Research Corporation, Littleton, Mass), using a modified Le Grand-El Hage unaccommodated theoretical eye model. The integrated wide-field SLO lens consists of 2 biconvex aspheric lenses and a 2-element convex-concave contact lens (Figure 1). It has $\times 0.23$ magnification and is afocal when used with gonioscopic gel. Antireflection coatings reduce reflections to less than $0.1 \%$ for $514-n m$ red-free and $835-n m$ infrared reflectance images. The integrated SLO wide-field contact lens system was used for all SLO wide-field contact lens images presented in this report.

Patients were examined with wide-field SLO angiography if a complete ophthalmic examination revealed an appropriate peripheral chorioretinal problem. The risks and benefits of all procedures were explained to each patient, and all gave informed consent before enrollment in the study. Each study patient underwent topical pharmacologic dilation and was positioned for imaging using a standard chin rest. Patients viewed a fixation target with their contralateral eye when feasible. After topical anesthesia, a wide-field SLO lens was placed with gonioscopic gel on the cornea of the eye to be examined. The examiner viewed the SLO's monitor, which displayed an inverted image of the patient's fundus produced by the wide-field contact lens system. Modified HRA Eye Explorer software, version 1.3 (Heidelberg Engineering $\mathrm{GmbH}$ ) permitted image inversion to facilitate ophthalmoscopy and angioscopy. The SLO focus was adjusted for maximum retinal vessel contrast. Proper lens alignment was maintained to optimize image quality.

Retinal angiography was performed by injecting a 5-mL solution of $25 \mathrm{mg}$ of ICG diluted with $5 \mathrm{~mL}$ of $20 \%$ sodium fluorescein. Although these are normal doses for conventional fundus cameras, they are higher than typical SLO doses, which are inadequate for wide-field imaging. Angiographic dye injection was followed by a $5-\mathrm{mL}$ isotonic sodium chloride solution flush. Dynamic, simultaneous fluorescein and ICG angioscopy movies were recorded at 6 frames per second and $256 \times 256$-pixel resolution. Recording began when ICG fluorescence first appeared on the SLO monitor and lasted for approximately 20 seconds. A series of 32 fluorescein and ICG images $(512 \times 512$ pixels, $1-\mathrm{mm}$ scan depth) were then recorded at different focal planes. Image collection typically required less than 2 minutes per eye. Patients with conditions such as diabetic retinopathy or central retinal vein occlusion received only sodium fluorescein ( $5 \mathrm{~mL}$ of $20 \%$ sodium fluorescein dye followed by a $5-\mathrm{mL}$ isotonic sodium chloride solution flush).

The HRA SLO's "compute mean" and "compute composite" software functions were used to average and process images from different focal planes, respectively. Analysis took approximately 15 seconds per eye using an $800-\mathrm{MHz}$ Pentium 4 computer system with $128 \mathrm{MB}$ of random access memory (Nima soluzioni informatiche SRL, Pessano con Bornago, Italy). The $512 \times 512$-pixel image files required 288 kilobytes of computer disk storage space. Processed images were stored as separate bitmap files in each patient's data folder for comparison with other images.

\section{RESULTS}

We used a wide-field SLO contact lens and an HRA SLO to evaluate more than 50 patients with diabetic retinopathy, chorioretinal tumors, retinal detachment, and other

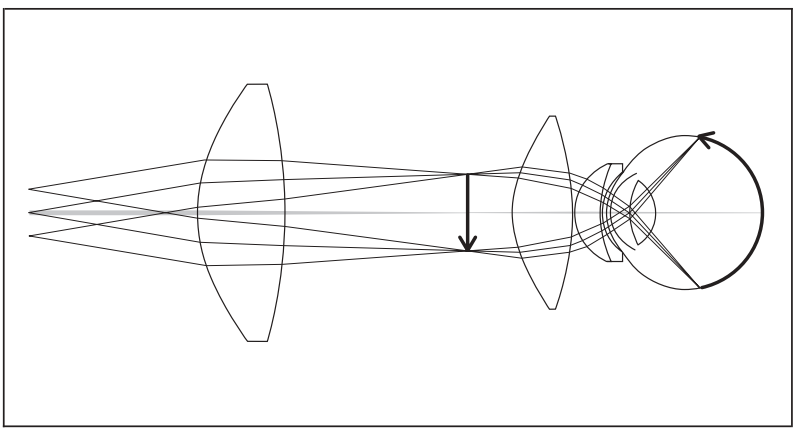

Figure 1. An integrated, multielement, wide-field contact lens system (Ocular Staurenghi 230 SLO Retina Lens; Ocular Instruments Inc, Bellevue, Wash). This system consists of 2 biconvex aspheric lenses and a 2-element convex-concave contact lens.

peripheral chorioretinal disorders. Figure 2 illustrates the $150^{\circ}, 100^{\circ}$, and $50^{\circ}$ imaging fields produced with HRA image field settings of $30^{\circ}, 20^{\circ}$, and $10^{\circ}$, respectively. Structures well anterior to the chorioretinal equator are imaged readily (Figures $\mathbf{3}, \mathbf{4}$, and 5). These figures also show that even more peripheral structures are detectable and recordable with changes in patient fixation.

The wide-field lens complements other previously documented advantages of SLO. ${ }^{2,3,9,11-13}$ The extended imaging field provided by the lens obviates the need for photomontages (Figure 4), avoiding their potential limitations, which include skipped areas and local variations in contrast and magnification. The peripheral retina and choroid are imaged readily through a small patient pupil, and iris neovascularization can be documented easily. The good vascular detail afforded by confocal SLO infrared reflectance imaging is unimpaired, as illustrated in Figure 5, which shows a varix of a vortex vein ampulla in its dilated and collapsed state.

Figures $\boldsymbol{6}, \mathbf{7}, \mathbf{8}, \boldsymbol{9}$, and $\mathbf{1 0}$ present the results of widefield SLO simultaneous fluorescein and ICG imaging. Figure 6 shows that the progression of brain tumor metastases is better delineated by fluorescein hyperfluorescent than ICG hypofluorescent patterns. Figure 7 illustrates that fluorescein and ICG images are each valuable in documenting capillary hemangioma closure and its hemodynamic sequelae. Figure 8 shows the local and regional consequences of successful cryopexy and scleral buckling repair of a retinal detachment. Figure 9 documents the chorioretinal sequelae of an acute nasal posterior ciliary artery occlusion, illustrating watershed topography and retrograde choroidal filling. Only an SLO movie can illustrate the dynamic nature of real-time, wide-field SLO angioscopy, which permits the identification and documentation of clinically relevant peripheral chorioretinal findings. Differences in fluorescein and ICG angiographic visualization of choroidal circulation are readily seen in the large region of the giant retinal tear in Figure 10.

\section{COMMENT}

Wide-field fluorescein angiograms can be created by (1) using a dedicated wide-field camera system, (2) using an accessory lens to expand the imaging field of a standard noncontact fundus camera or SLO, or (3) pro- 


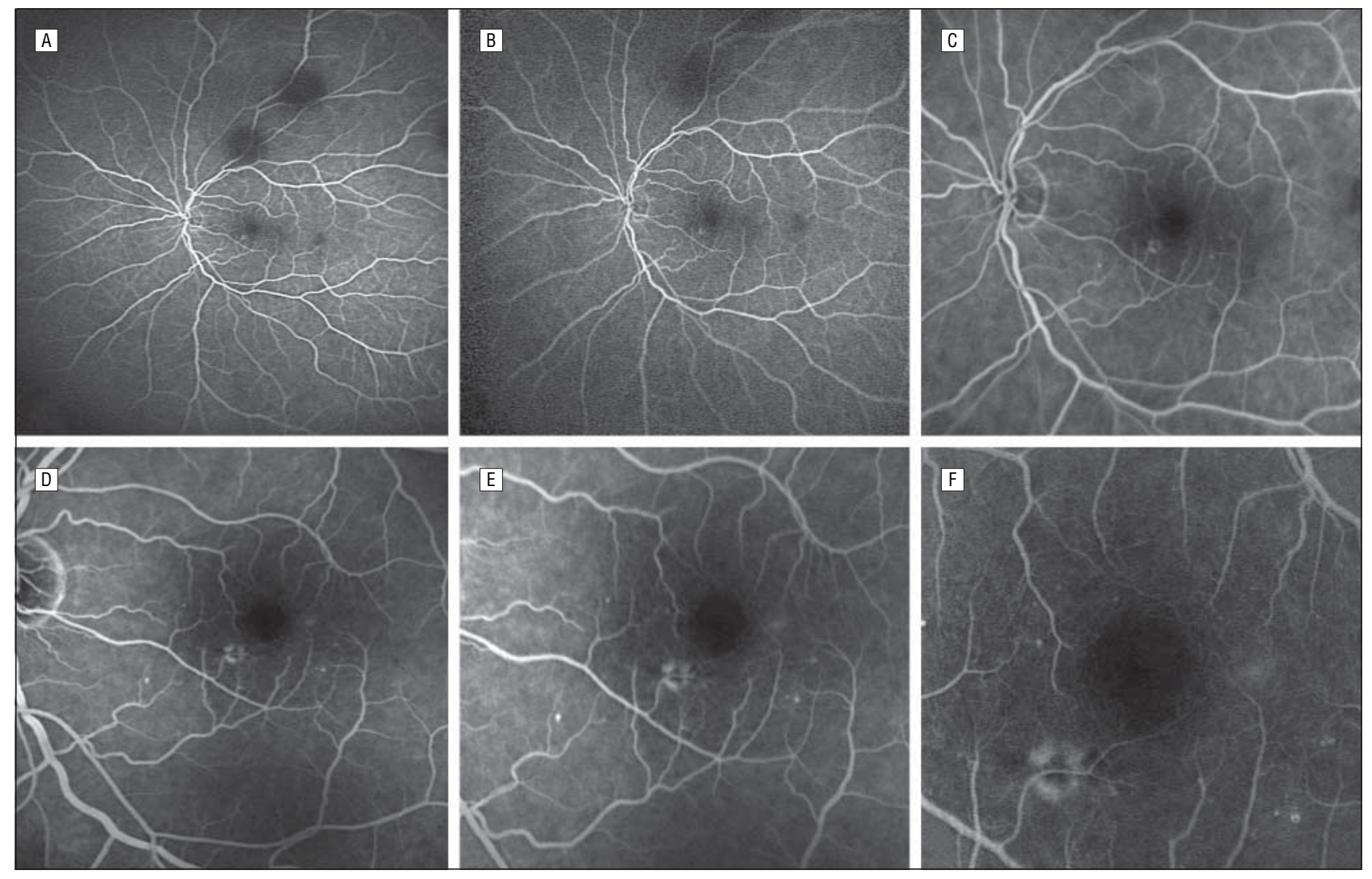

Figure 2. Fluorescein angiographic images of a 55-year-old man with nonproliferative diabetic retinopathy. The wide-field scanning laser ophthalmoscope (SLO) lens provides image fields of $150^{\circ}(\mathrm{A}), 100^{\circ}(\mathrm{B})$, and $50^{\circ}(\mathrm{C})$ for SLO imaging fields of $30^{\circ}(\mathrm{D}), 20^{\circ}(\mathrm{E})$, and $10^{\circ}(\mathrm{F})$, respectively. The hypofluorescent regions in the superior-temporal midperiphery in $\mathrm{A}$ and $\mathrm{B}$ are artifacts due to vitreous opacities or air bubbles in gonioscopic gel.

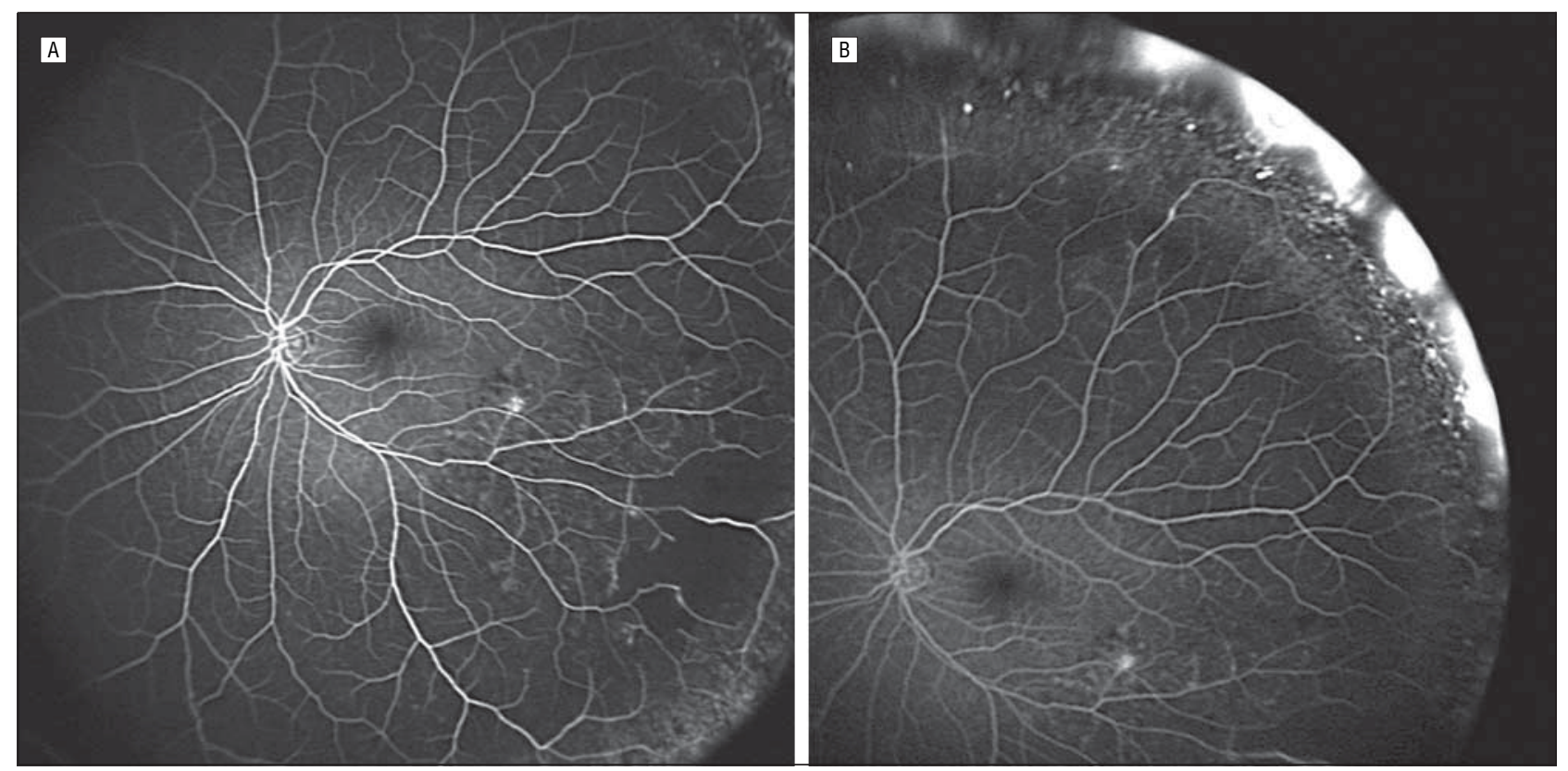

Figure 3. Fluorescein angiographic images of a 38-year-old man with Behçet disease. Wide-field scanning laser ophthalmoscope (SL0) angiography reveals areas of capillary nonperfusion and extensive retinal neovascularization well beyond the imaging fields of conventional fundus cameras and SLOs.

ducing a photomontage from a conventional fundus camera or SLO images. Each method has its advantages and disadvantages.

Photomontages of static images can be produced manually or by computer automation. Manual photomon- tages are created by cutting and pasting photographic prints or by overlapping and blending their digitized images with photo-editing software. ${ }^{14-16}$ Higher resolution is achievable with wide-field manual photomontages ${ }^{17,18}$ but their preparation is lengthy and laborious. 

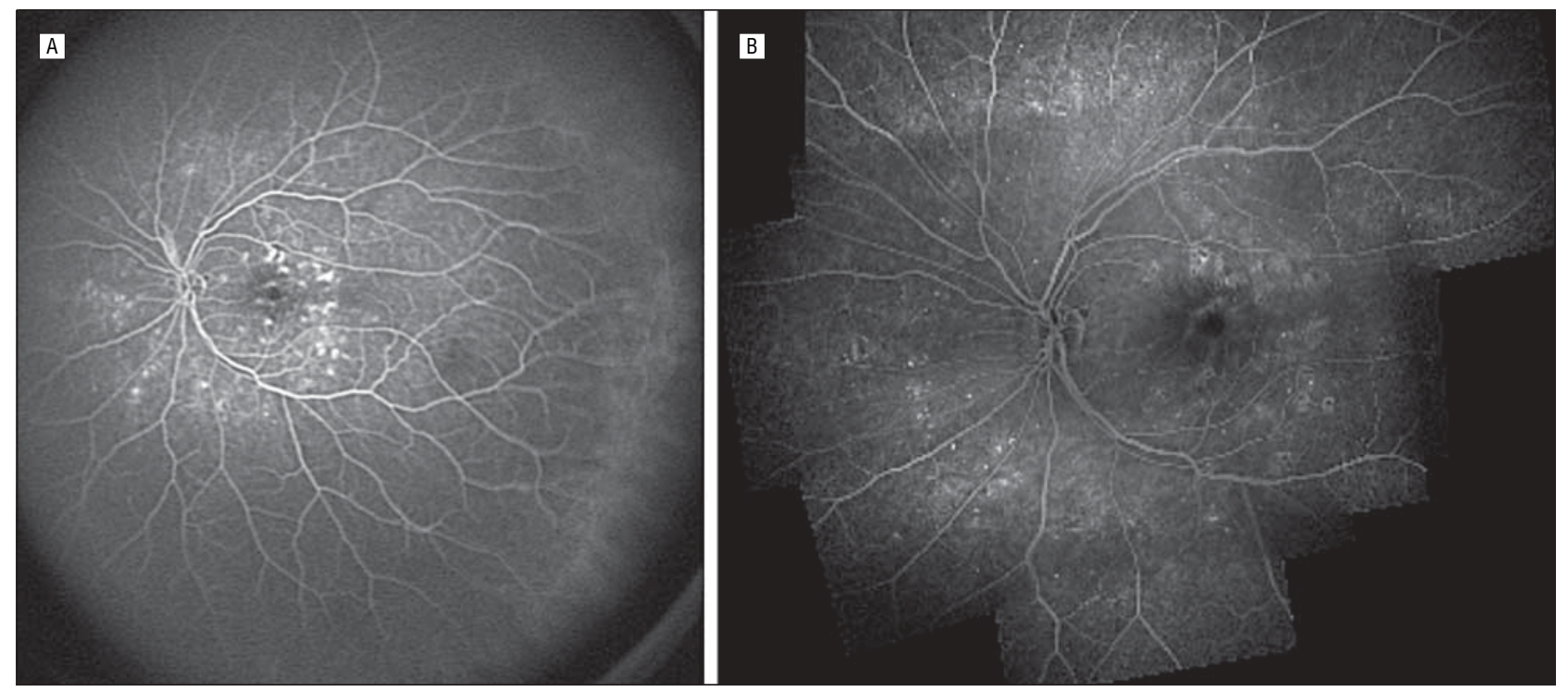

Figure 4. Fluorescein angiographic images of a 56 -year-old diabetic man using the $30^{\circ}$ image setting of the Heidelberg retina angiograph (HRA; Heidelberg Engineering GmbH, Dossenheim, Germany). A, A single wide-field scanning laser ophthalmoscope (SLO) lens image. B, A computer-generated photomontage of individual images using HRA Eye Explorer software (Heidelberg Engineering $\mathrm{GmbH}$ ). The photomontage provides valuable integration of individual images, but skipped areas are present, and resolution and image field size are lower than those of a wide-field SLO lens image.
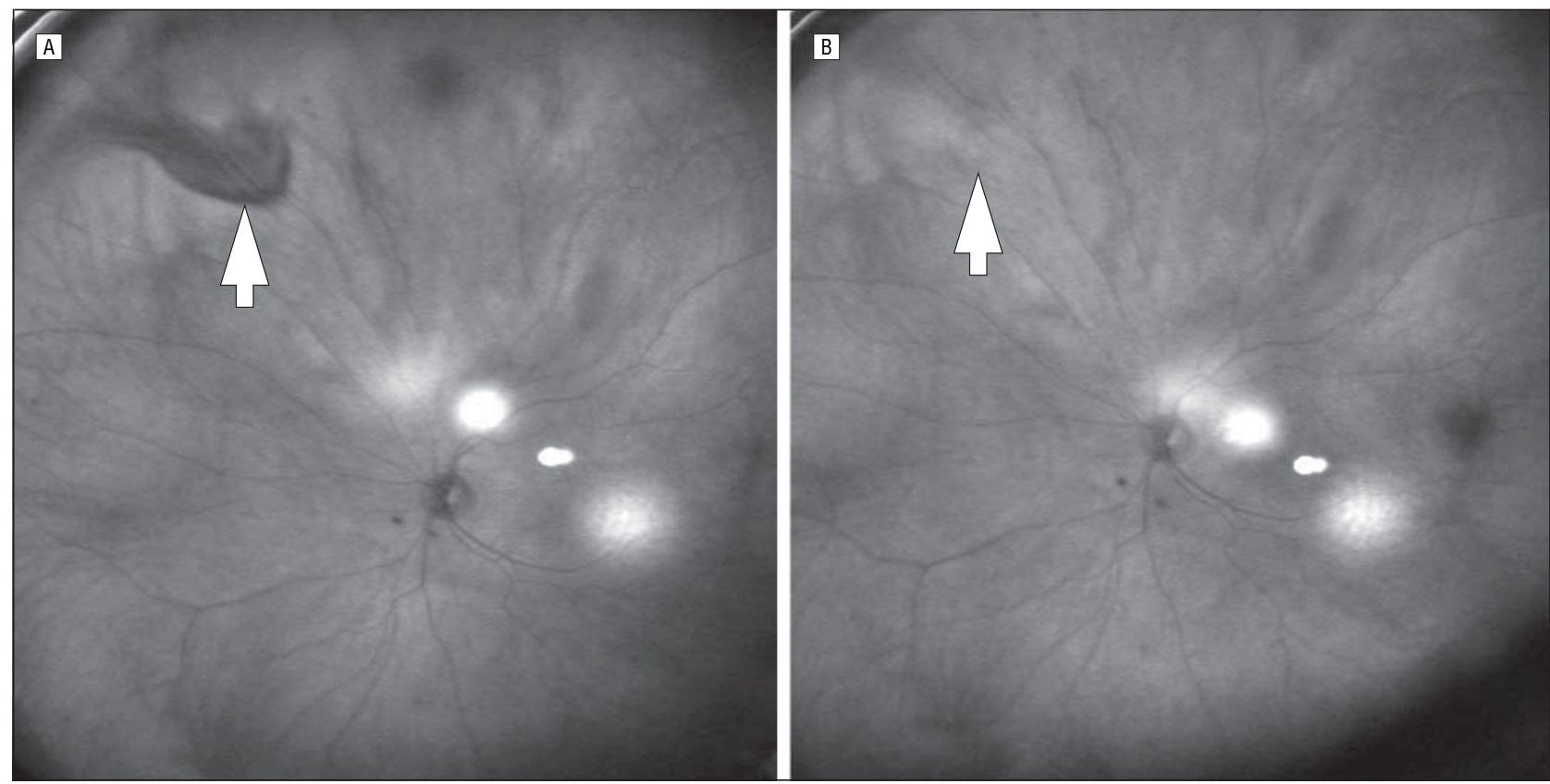

Figure 5. The wide-field scanning laser ophthalmoscope (SLO) lens can be used without angiography dyes for infrared fundus photography. The arrows identify an asymptomatic varix of a vortex vein ampulla in an 835-nm wide-field SLO reflectance image of the left eye of a 64-year-old woman. The varix is shown in its dilated (A) and collapsed (B) states. Lens reflections suppressed by angiography filters are apparent centrally in these infrared reflectance images.

The HRA Eye Explorer software automates photomontage preparation, producing photomontages that range from $100^{\circ}$ to $140^{\circ} .{ }^{19}$ The software takes approximately 5 minutes to identify and align retinal vascular patterns in adjacent images. The additional photography required to produce a photomontage requires only a few minutes for an experienced photographer.

Manual and automated photomontages have excellent image detail in their individual small-field images, but neither is useful for dynamic angiography. Both techniques introduce inaccuracies in judging the extent of or changes in a large peripheral chorioretinal structure because (1) contrast and brightness can vary across photomontages, (2) magnification and distortion can vary within and between photomontage elements that represent different 2-dimensional projections of the highly curved peripheral retina, and (3) automated processes can produce skipped areas (Figure 4). Dynamic angioscopy, changes in patient fixation, and the broad $150^{\circ}$ SLO imaging field provided by a wide-field SLO contact lens system obviate the need for manual or computerautomated photomontages.

Several dedicated wide-field camera systems are available currently. A 2-color, very wide-field SLO has been 

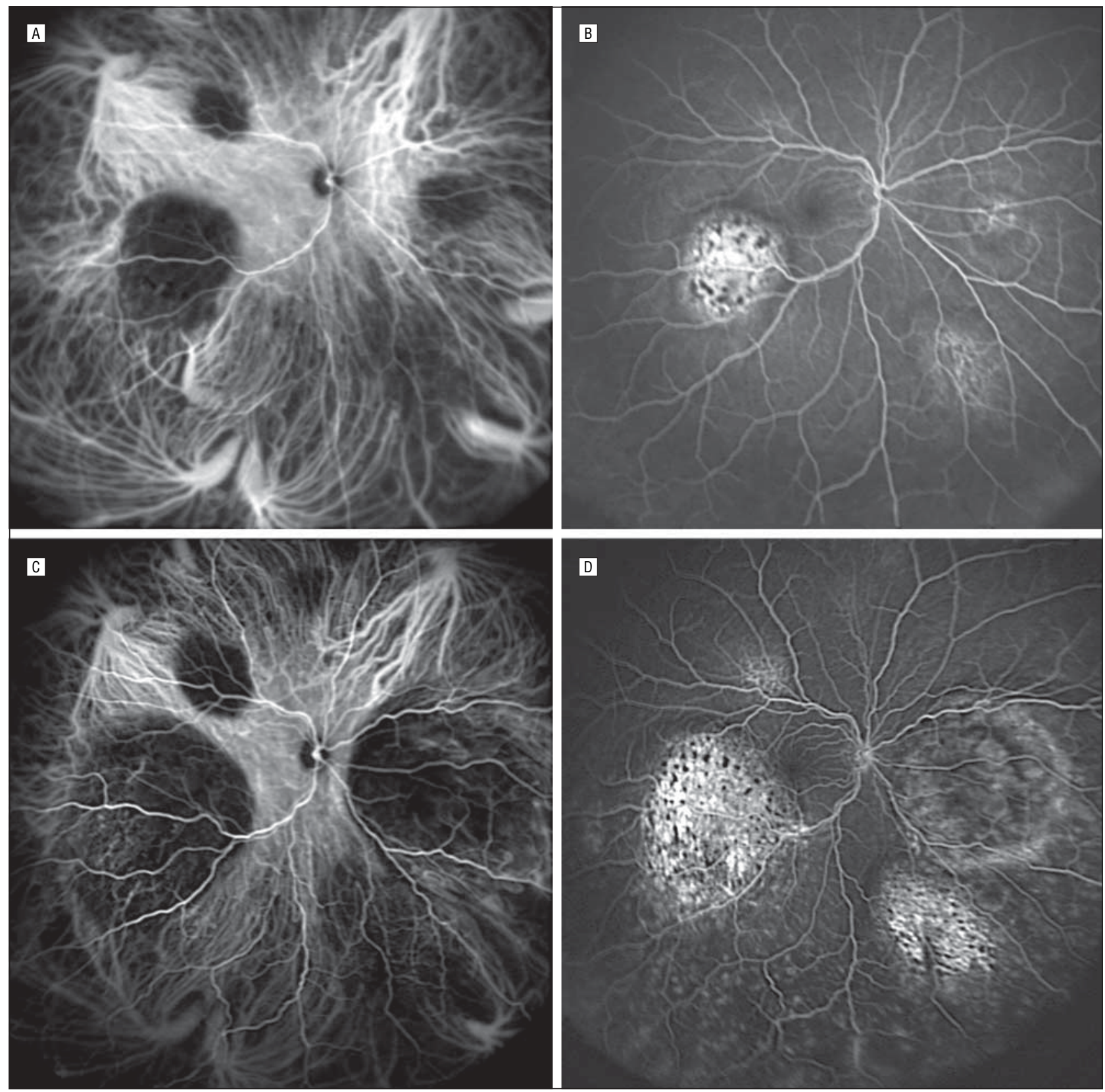

Figure 6. Wide-field scanning laser ophthalmoscope (SLO) images document the progression of brain tumor metastases in a 43-year-old woman. A and C, Indocyanine green images. B and D, Fluorescein images. The images in C and D were taken 12 months after the images in A and B.

developed for retinal examination (Panoramic200; Optos plc, Dunfermline, Scotland) with lower resolution than angiographic SLOs. Non-SLO, wide-field camera systems use a contact ophthalmoscopic lens and either transscleral illumination primarily for adult patients (Panoret 1000; Medibell Ltd, Haifa, Israel) or transpupillary illumination primarily for pediatric patients (Retcam 120; Massie Research Laboratories Inc, Dublin, Calif). The original Equator-plus camera used either type of illumination ${ }^{\top}$ and produced static, low-magnification, $148^{\circ}$ film images.

Contemporary wide-field contact cameras are independent, computer-automated imaging systems that offer dynamic, $90^{\circ}$ to $110^{\circ}$ digital images with higher resolution than the original Equator-plus camera. They produce excellent wide-field images, but since all available retinal detail is in the ophthalmoscopic image produced by their contact ophthalmoscopy lens, ${ }^{20,21}$ magnifying that image optically and/or digitally cannot provide the additional detail available in high-resolution, smallerfield fundus camera or SLO images.

Accessory contact and noncontact lenses have been used to increase the field of view of conventional fundus cameras. ${ }^{4-6}$ The feasibility of increasing the imaging field of the first nonconfocal SLO was demonstrated using the contact lens element of an Equator-plus camera. ${ }^{9}$ A handheld, noncontact 30-D ophthalmoscopy lens was used to increase the imaging field of a confocal SLO (Rodenstock $\mathrm{GmbH}$, Munich, Germany) from $40^{\circ}$ to $70^{\circ}$ to study the peripheral choroid's watershed zone. ${ }^{10}$ Place- 

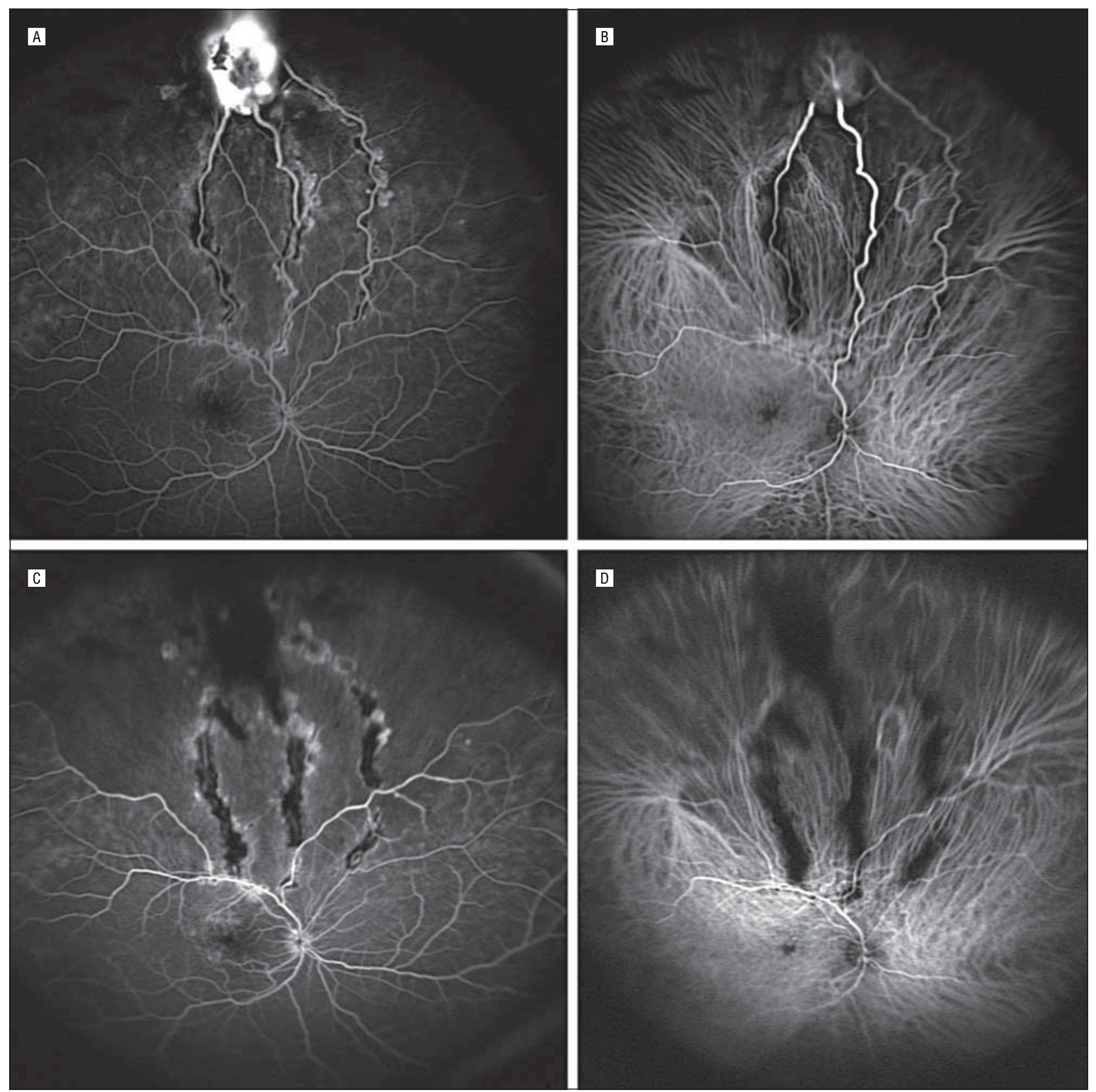

Figure 7. Wide-field scanning laser ophthalmoscope images of a 24-year-old woman with macular exudate from a capillary hemangioma in the superior retinal periphery of her right eye. A and C, Fluorescein images. B and D, Indocyanine green images. Incomplete laser photocoagulation closure of the hemangioma's central draining venule and its 2 flanking feeding arterioles is seen. Capillary hemangioma closure was documented by the absence of perfusion in dynamic wide-field and higher-resolution smaller-field images. C and D, Complete closure of those vessels and the hemangioma's circulation after additional laser photocoagulation are evident. Macular exudates resolved, but retinal circulation is closed superiorly where choroidal perfusion is preserved.

ment of the wide-field SLO lens on a patient's cornea requires a few seconds. None of our patients had corneal abrasions, which can occur in any contact lens procedure.

The images presented in Figures 2 through 10 demonstrate the clinical value of a wide-field SLO contact lens and show that a confocal SLO has adequate resolution for detailed reflectance and angiographic imaging even when an accessory lens produces a 5 -fold increase in its imaging field. The wide-field SLO lens can be used with adults or children, although the contact element is too large for infants. It is also useful for patients with aphakic and pseudophakic eyes, and for patients with cataracts of low to moderate opacity. It provides good images with small pupils, although field size is not as large as with dilated pupils. We were unable to perform autofluorescence imaging with the wide-field SLO lens because of its insufficient overall optical transmission.

Local and global chorioretinal features can be documented with the wide-field SLO lens system by "zooming in" on selected structures using smaller SLO field settings. Higher SLO resolutions are available when needed by removing the contact lens, irrigating the cornea (ide- 

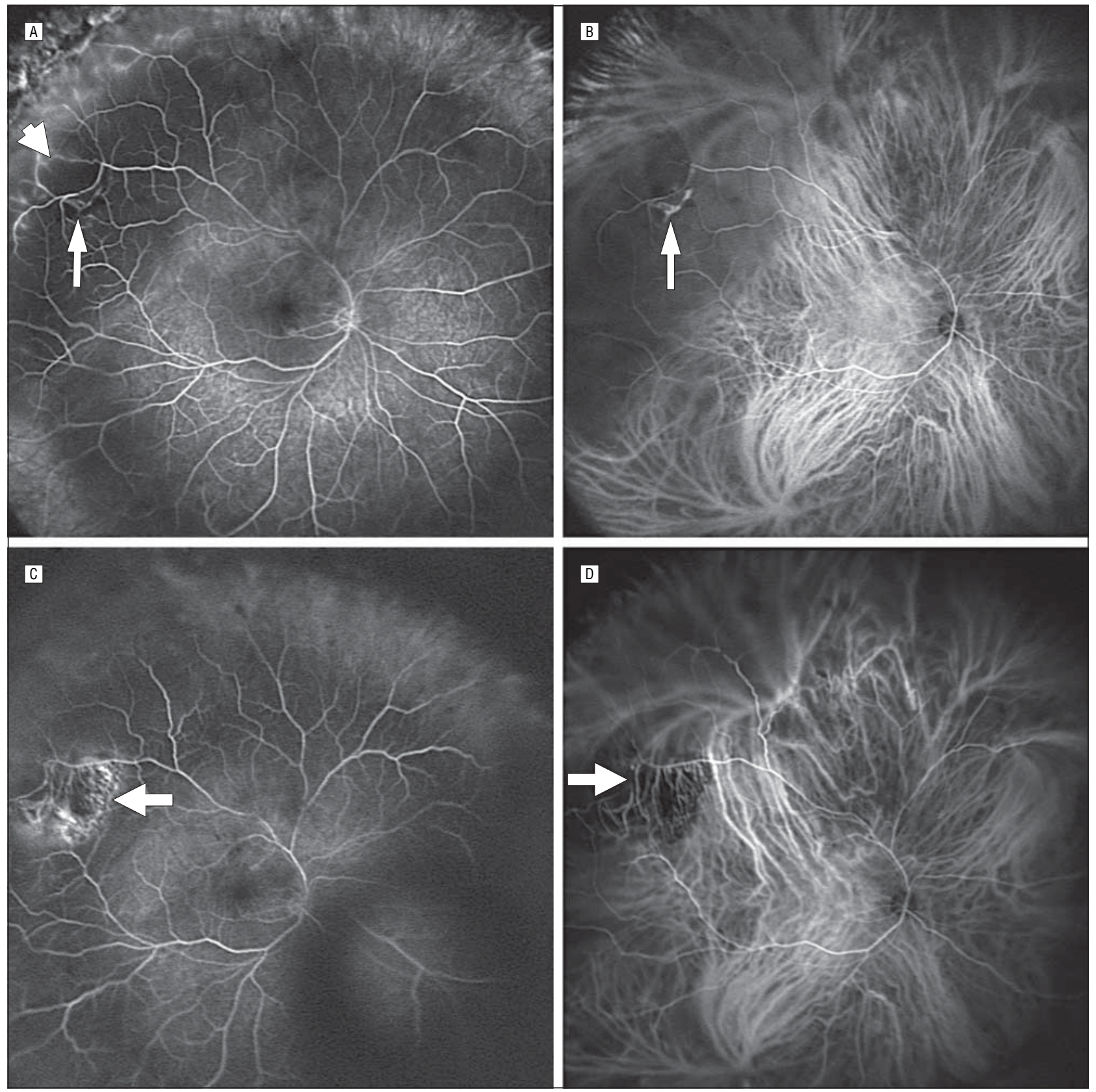

Figure 8. Wide-field scanning laser ophthalmoscope images of a pseudophakic eye in a 58-year-old man. Images were taken before (A and B) and after (C and D) cryopexy and scleral buckling retinal detachment surgery. Vertical white arrows show the location of the retinal break. The arrowhead in A identifies the ischemic retinal flap. Horizontal arrows identify the cryopexy site. Detached temporal and superior-temporal peripheral retina in the right eye are better delineated in the indocyanine green (ICG) (B) than the fluorescein (A) image because of a prominent annular midperipheral hypofluorescent artifact in fluorescein angiogram frames due to this patient's intraocular lens. A prominent artifact in the inferior-nasal retina of the right eye due to a gonioscopic gel air bubble is also much more prominent in fluorescein (C) than ICG (D) images. No angiographically apparent vascular changes overlie the scleral buckle after retinal reattachment (C and D), but the retinal pigment epithelium and choriocapillaris are disrupted at the cryopexy site, where larger choroidal vessels are preserved.

ally with a 5\% glucose solution), and using the SLO's native $30^{\circ}, 20^{\circ}$, or $10^{\circ}$ image field sizes. Reflections during fluorescein and ICG angiography are negligible because of the SLO's excellent angiography filters, but lens reflections can be seen in the infrared fundus photography shown in Figure 5. Good resolution is preserved in late angiogram frames.

Figures 6 through 10 demonstrate the clinical value of wide-field simultaneous fluorescein and ICG SLO angiography. Areas of peripheral chorioretinal nonperfusion and neovascularization can be detected and documented well beyond the range of conventional fundus cameras. Wide-field peripheral retinal and highresolution foveal angioscopy can be performed and documented in the same study. Indirect ophthalmoscopy findings can be confirmed, recorded, and analyzed. Vortex veins can be localized for guiding subsequent vitreoretinal surgery. Scanning laser ophthalmoscope fields comparable to those of wide-field contact laser lenses ${ }^{21}$ can be recorded to facilitate photocoagulation. The wide-field SLO lens increases the utility of high-resolution SLO angiography and provides convenient static and dynamic re- 

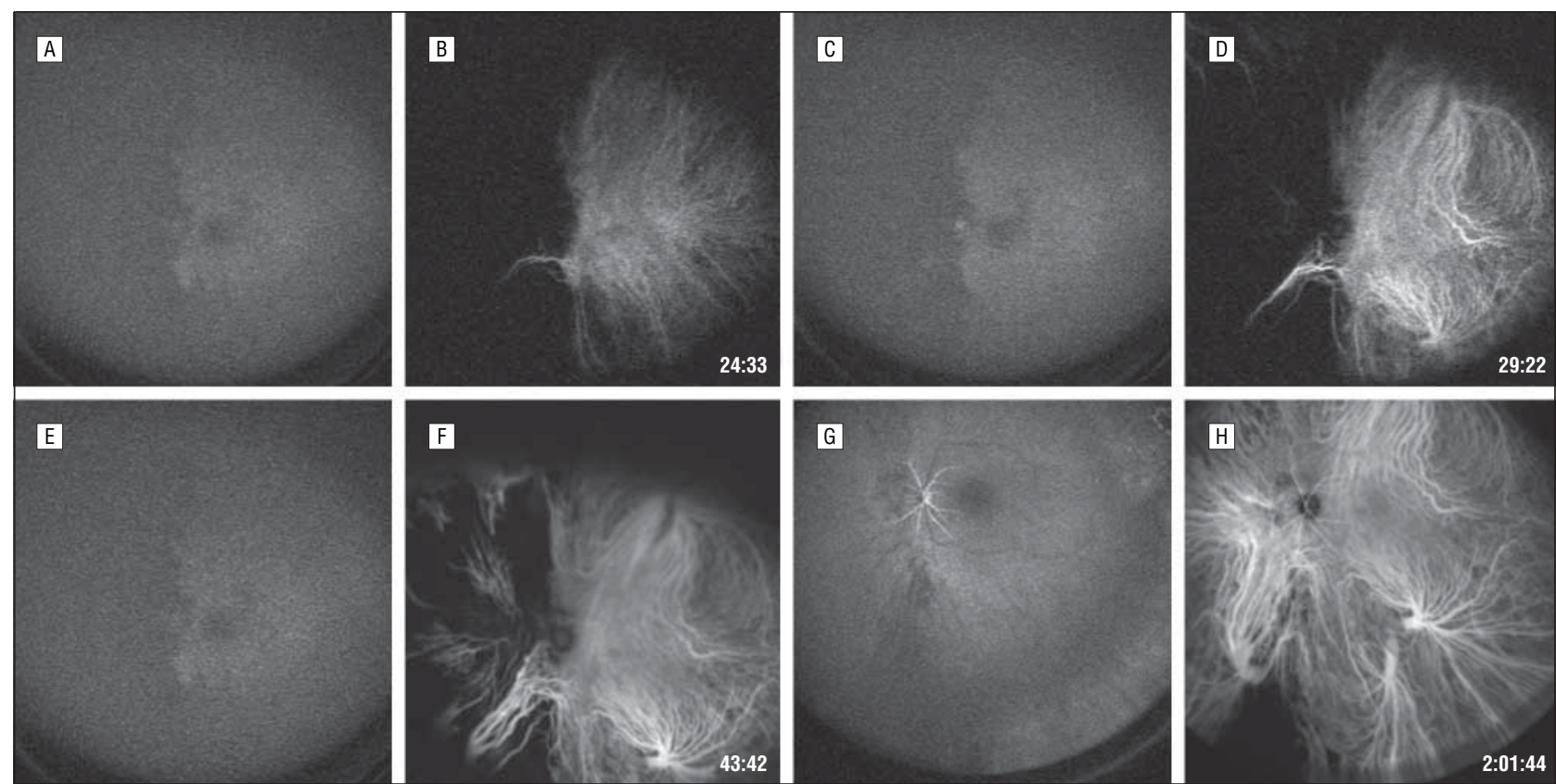

Figure 9. Wide-field scanning laser ophthalmoscope angiographic images of a 54-year-old man after an acute nasal posterior ciliary artery occlusion. Fluorescein angiograms ( $A, C, E$, and $G$ ) show that retinal vascular filling and transit are markedly delayed and diminished. The indocyanine green (ICG) images (B, D, F, and $\mathrm{H})$ document peripheral retrograde choroidal arteries ${ }^{10}$ in the nasal choroid and a watershed zone between regions subserved by those vessels. Regions of faint fluorescein hyperfluorescence correspond to regions of choroidal perfusion as documented in the ICG images. Time signatures in B, D, F, and $H$ mark time after injection.
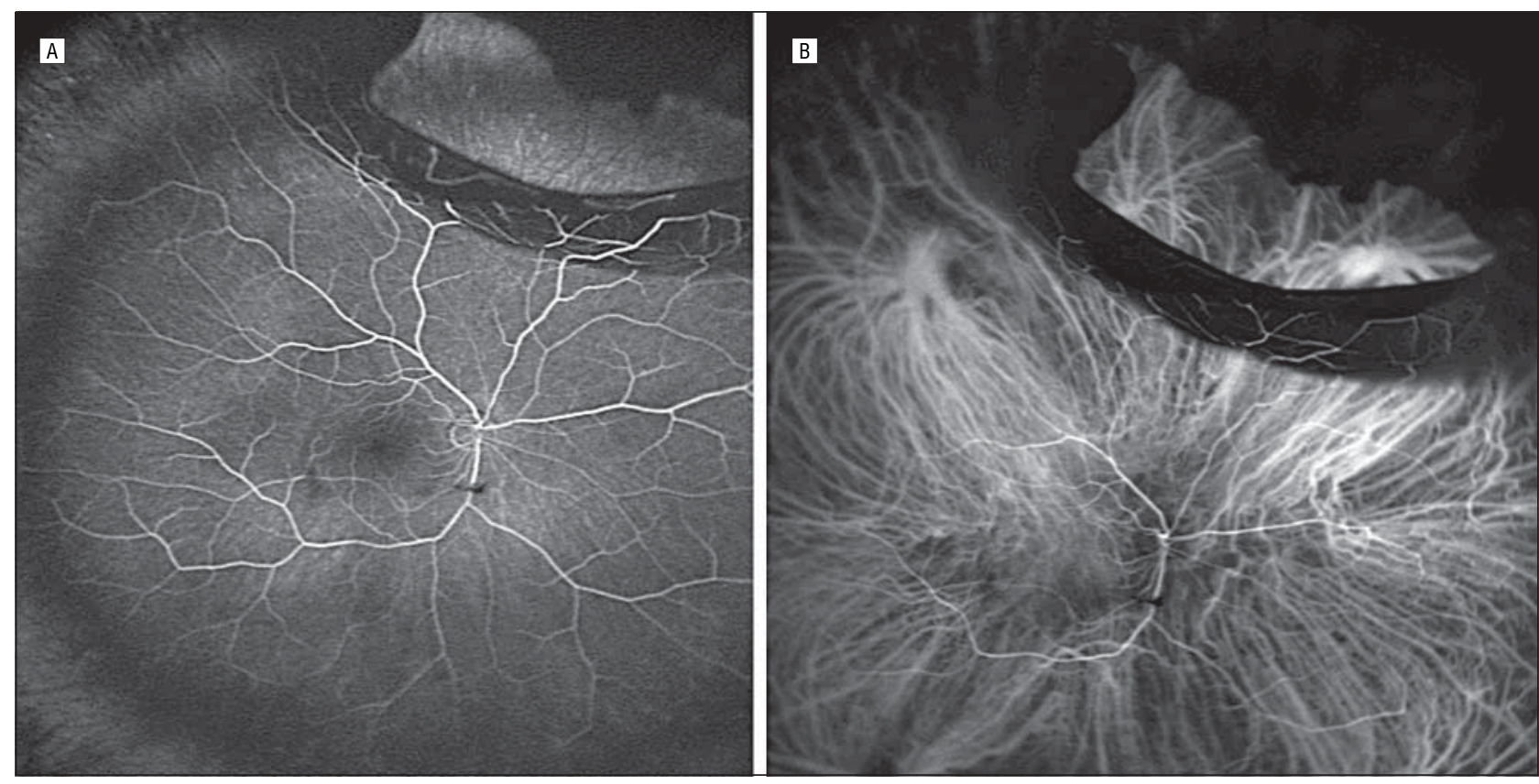

Figure 10. Wide-field scanning laser ophthalmoscope fluorescein and indocyanine green (ICG) images of a 54-year-old man with a giant retinal tear. The ICG image (B) shows that choroidal perfusion is preserved anterior to the tear. The fluorescein image (A) shows that retinal pigment epithelium anterior to the break hinders visualization of underlying choroidal circulation even in the absence of overlying neural retina. This obstruction is due largely to melanin's increased absorption of optical radiation at shorter fluorescein than longer ICG angiography wavelengths. Choroidal vessels underlying the tear itself are not visible in fluorescein or ICG angiography, because they are obscured by the tangentially oriented overlying flap and rolled edge of edematous detached neural retina. The hypofluorescent spots inferior to the optic disc and along the inferior-temporal arcade are low-lying vitreous hemorrhages.

cords of the vascular structure and hemodynamics of the peripheral retina and choroid.

Submitted for Publication: July 17, 2003; final revision received June 10, 2004; accepted June 29, 2004.
Correspondence: Giovanni Staurenghi, MD, Department of Ophthalmology, University of Brescia, Via Tiraboschi, 8, 20135 Milan, Italy (giovanni.staurenghi@unimi.it). Previous Presentations: Some of this work has been presented previously as posters 2511 and 2999, respec- 
tively, at the 2002 and 2003 Annual Meetings of the Association for Research in Vision and Ophthalmology, Fort Lauderdale, Fla.

\section{REFERENCES}

1. Webb RH, Hughes GW, Delori FC. Confocal scanning laser ophthalmoscope. App/ Opt. 1987;26:1492-1499.

2. Freeman WR, Bartsch DU, Mueller AJ, Banker AS, Weinreb RN. Simultaneous indocyanine green and fluorescein angiography using a confocal scanning laser ophthalmoscope. Arch Ophthalmol. 1998;116:455-463.

3. Bartsch DU, Weinreb RN, Zinser G, Freeman WR. Confocal scanning infrared laser ophthalmoscopy for indocyanine green angiography. Am J Ophthalmol. 1995; 120:642-651.

4. Noyori KS, Chino K, Deguchi T. Wide field fluorescein angiography by use of contact lens. Retina. 1983;3:131-134.

5. Ozerdem U, Freeman WR, Bartsch DU, Clark TM. A simple noncontact wideangle fundus photography procedure for clinical and research use. Retina. 2001; 21:189-190.

6. Spaide RF, Orlock DA, Herrmann-Delemazure B, et al. Wide-angle indocyanine green angiography. Retina. 1998;18:44-49.

7. Pomerantzeff 0. Equator-plus camera. Invest Ophthalmol. 1975;14:401406.

8. Roth DB, Morales D, Feuer WJ, Hess D, Johnson RA, Flynn JT. Screening for retinopathy of prematurity employing the Retcam 120 : sensitivity and specificity. Arch Ophthalmol. 2001;119:268-272.

9. Mainster MA, Timberlake GT, Webb RH, Hughes GW. Scanning laser ophthalmoscopy: clinical applications. Ophthalmology. 1982;89:852-857.
10. Takahashi K, Muraoka K, Kishi S, Shimizu K. Watershed zone in the human peripheral choroid. Ophthalmology. 1996;103:336-342.

11. Elsner AE, Burns SA, Weiter JJ, Delori FC. Infrared imaging of sub-retinal structures in the human ocular fundus. Vision Res. 1996;36:191-205.

12. Staurenghi G, Aschero M, La Capria A, Gonnella P, Orzalesi N. Visualization of neovascular membranes with infrared light without dye injection by means of a scanning laser ophthalmoscope. Arch Ophthalmol. 1996;114:365.

13. Holz FG, Bellmann C, Dithmar S, Rohrschneider K, Burk RO, Volcker HE. Simultaneous fluorescein and indocyanine green angiography with a confocal laser ophthalmoscope. Ophthalmologe. 1997;94:348-353.

14. Clark TM, Freeman WR, Goldbaum MH. Digital overlay of fluorescein angiograms and fundus images for treatment of subretinal neovascularization. Retina. 1992;12:118-126.

15. Sleightholm MA, Arnold J, Aldington SJ, Kohner EM. Computer-aided digitisation of fundus photographs. Clin Phys Physiol Meas. 1984;5:295-301.

16. Mahurkar AA, Vivino MA, Trus BL, Kuehl EM, Datiles MB III, Kaiser-Kupfer MI Constructing retinal fundus photomontages: a new computer-based method. Invest Ophthalmol Vis Sci. 1996;37:1675-1683.

17. Shimizu K, Kobayashi Y, Muraoka K. Midperipheral fundus involvement in diabetic retinopathy. Ophthalmology. 1981;88:601-612.

18. Niki T, Muraoka K, Shimizu K. Distribution of capillary nonperfusion in earlystage diabetic retinopathy. Ophthalmology. 1984;91:1431-1439.

19. Rivero ME, Bartsch DU, Otto T, Freeman WR. Automated scanning laser ophthalmoscope image montages of retinal diseases. Ophthalmology. 1999;106: 2296-2300.

20. Pomerantzeff 0 , Webb RH, Delori FC. Image formation in fundus cameras. Invest Ophthalmol Vis Sci. 1979;18:630-637.

21. Mainster MA, Reichel E, Harrington PG, Erickson PJ, Graham RD. Ophthalmoscopic contact lenses for transpupillary thermotherapy. Semin Ophthalmol. 2001; 16:60-65. 